\title{
LA INFLUENCIA DE LA CORRUPCIÓN SOBRE EL DERECHO A UNA EDUCACIÓN DE CALIDAD. UN ESTUDIO DE CORRELACIÓN
}

\author{
José Francisco Báez Corona* \\ Jaqueline del C. Jongitud Zamora**
}

Fecha de recibido: 26 de noviembre de 2013.

Fecha de aprobado: 31 de marzo de 2014.

Artículo de Reflexión

Forma de citación: Báez, J.F. \& Jongitud, J.C. (2014). La influencia de la corrupción sobre el derecho a una educación de calidad. Un estudio de correlación. Revista Prolegómenos. Derechos y Valores, 17, 33, 123-142

\begin{abstract}
Resumen
El estudio aborda la relación entre percepción de la corrupción y el derecho a una educación básica de calidad desde un punto de vista teórico y estadístico, lo anterior, con base en la correlación del Índice de Percepción de la Corrupción de Transparencia Internacional y la prueba del Programa para la Evaluación Internacional de los Estudiantes de la Organización para la Cooperación y el Desarrollo Económico. El análisis del estado del arte sobre la relación entre estas variables y los resultados del coeficiente de correlación, entre otras herramientas estadísticas, muestran que existe una alta relación: a menor percepción de la corrupción en un país corresponden mejores resultados en la evaluación de su calidad educativa.
\end{abstract}

\section{Palabras clave:}

Percepción de la corrupción; derecho a la educación de calidad; educación básica; Índice de Percepción de la Corrupción (IPC); Programa para la Evaluación Internacional de Estudiantes (PISA).

* Doctor en Derecho Público, maestro y especialista en Docencia Universitaria, licenciado en Derecho y en Pedagogía, Miembro del Sistema Nacional de Investigadores del Consejo Nacional de Ciencia y Tecnología en México, Investigador del Instituto de Investigaciones Jurídicas de la Universidad Veracruzana, autor y coordinador de diversas publicaciones nacionales e internacionales, especialista en el análisis de temas jurídicos con un enfoque transdisciplinar. (Calle Azueta . 188bis, Zona Centro, Xalapa, Veracruz, México, CP. 91000. Tel. 0052 228814.16.82, baez.corona@gmail.com)

** Doctora en Derecho por la Universidad Veracruzana, investigadora del Instituto de Investigaciones Jurídicas de la misma Casa de Estudios y miembro del Sistema Nacional de Investigadores del Consejo Nacional de Ciencia y Tecnología en México, realizó estancia postdoctoral en la Universidad de Valencia, España. (Calle Hermenegildo Galeana esquina 7 de Noviembre S/N, Zona Centro, Xalapa, México, CP. 91000. Tel. 0052 228818.68.41, jakinakauma@hotmail.com) 


\title{
THE INFLUENCE OF CORRUPTION ON THE RIGHT TO A QUALITY EDUCATION. A CORRELATION STUDY
}

\begin{abstract}
The study addresses the relation between the perception of corruption and the right to a quality basic education from a theoretical and statistical standpoint, the latter based on the correlation of the Corruption Perceptions Index of Transparency International and the Programme for International Student Assessment test of the Organisation for Economic Co-operation and Development. The state of the art analysis on the relationship between these variables and the results of the correlation coefficient, among other statistical tools, show that there is a strong relationship: the lower the perception of corruption in a country is the better are the results of its educational quality evaluation.
\end{abstract}

\section{Keywords:}

Corruption perception, Right to a quality education, Basic education, Corruption Perceptions Index (CPI), Programme for International Student Assessment (PISA)

\section{A INFLUÊNCIA DA CORRUPÇÃO NA DIREITO A UMA EDUCAÇÃO DE QUALIDADE. UM ESTUDO DE CORRELAÇÃO}

\begin{abstract}
Resumo
O estudo aborda a relação entre a percepção da corrupção e do direito a uma educação básica de qualidade a partir de um ponto de vista teórico e estatístico, este último com base na correlação das Percepções de Corrupção da Transparência Internacional Índice eo Programa para teste de Avaliação Internacional de Estudantes da Organização para a Cooperação e Desenvolvimento Econômico. O estado da arte sobre a análise da relação entre estas variáveis e os resultados do coeficiente de correlação, entre outras ferramentas estatísticas, mostram que há uma forte relação: quanto menor a percepção de corrupção em um país é o melhor são os resultados da sua avaliação da qualidade educacional.
\end{abstract}

\section{Palavras-Chave:}

Percepção da Corrupção, Direito a uma educação de qualidade, Educação básica, Corrupção Perceptions Index (CPI), Programa de Avaliação Internacional de Estudantes (PISA).

\section{A MODO DE INTRODUCCIÓN, CORRELACIÓN ENTRE CORRUPCIÓN Y EDUCACIÓN: PLANTEAMIENTO METODOLÓGICO}

Corrupción y educación son variables que pueden ser analizadas de forma independiente; sin embargo, también hay estudios $y$ antecedentes que las han vinculado, tanto entre sí como con respecto a otros indicadores. (Stephen, 2004, p. 637-348)

No obstante, la mayoría de los estudios precedentes se concretan en plantear su relación desde una base solo teórica, por lo cual en esta investigaciónsebuscóconocer desde un enfoque 
estadístico la correspondencia entre estas variables, cuestionando si existe influencia de la corrupción sobre el derecho a una educación de calidad, a partir de la pregunta: ¿cuál es la correlación estadística entre la percepción de corrupción y la educación de calidad, conforme a los indicadores internacionales: índice de percepción de la corrupción (IPC) y los resultados del Programa para la Evaluación Internacional de Estudiantes (PISA) en el 2009. El estado del arte sobre el tema permitió formular la hipótesis de que: «a menor percepción de la corrupción en un país corresponden mejores resultados en la evaluación de su calidad educativa» o en forma más concreta: a mejor calificación de IPC corresponderá un mejor resultado en las pruebas PISA.

Por lo anterior, la investigación tuvo como objetivo general determinar estadísticamente si existe una correlación entre percepción de la corrupción y el derecho a una educación de calidad, conforme a los indicadores internacionales IPC y PISA.

Como justificación, se consideró que el alcanzar el objetivo y demostrar la hipótesis planteada, permitiría reforzar desde una metodología estadística los argumentos teóricos existentes sobre la incidencia de la corrupción en diferentes esferas de la vida pública y, particularmente, en cuanto a la educación de calidad como un derecho humano, situación que evidenciaría y podría constituir la base de otras investigaciones referentes a la necesidad del reforzamiento de los marcos normativo, político y social de combate a la corrupción.

Para ello, se utilizaron las herramientas estadísticas «coeficiente de correlación de Pearson», la prueba de Kruskal Wallis, el coeficiente de determinación, así como técnicas documentales que permitieron la descripción de estudios que vinculan a la corrupción con diversos factores como la vulneración de derechos humanos $(\mathrm{DDHH})$, la desigualdad social, el rezago en materia de desarrollo humano o el derecho a la educación, asimismo, se desarrollaron los conceptos clave del estudio.
Por otra parte, las medidas del IPC y PISA fueron seleccionadas por las siguientes razones:

- Se trata de dos indicadores de corrupción y educación, respectivamente, que gozan de amplia aceptación, así como de validez interna en sus resultados.

- Son indicadores sobre los cuales se cuenta con información para diferentes países en el mundo, que representan a todos los continentes.

- Los datos utilizados fueron de 2009, ya que al momento de realizar esta investigación este fue el año más reciente y en el que se cuenta con ambas medidas, puesto que el IPC se publica anualmente, pero la prueba PISA solo se realiza cada tres años.

A partir de lo anterior, en el estudio se incluyó una muestra determinada estadísticamente $e^{1} \mathrm{de}$ 65 países, los cuales fueron seleccionados por cumplir con los siguientes criterios:

Inclusión. Países que participaron para ambos estudios, IPC y PISA en 2009.

Exclusión. Países que no participaron en ninguna o participaron en solo una de las pruebas, así como países que fueron considerados de manera diferente para una prueba o la otra de acuerdo a su conformación política. ${ }^{2}$

1 Para determinar el tamaño de la muestra se utilizó un muestreo aleatorio simple Empleando la fórmula siguiente:

$n$ : es el tamaño de la muestra;

$Z$ : es el nivel de confianza;

$p$ : es la variabilidad positiva;

$q$ : es la variabilidad negativa también representada por (1-p);

$N$ : es el tamaño de la población;

E: es el error estándar.

Considerando en el tamaño de la población $\mathrm{N}=180$ países $y$ en vista de que no existen antecedentes de un estudio previo a este tipo para obtener la proporción, se empleó máxima varianza, es decir el $50 \%(p=0.5)$, con un nivel de confianza de $95 \%$ y un error estándar del $10.55 \%$, con lo se obtuvo un tamaño mínimo de muestra de $=59$ países. La lista de países participantes se amplió de 59 a 65 al aplicar los criterios de inclusión y exclusión. (Bosch, 2005).

2 Ejemplo de algunos países excluidos por los criterios: 
A continuación, se presenta para cada una de las variables que integra el estudio, percepción de la corrupción y resultados de la evaluación de la calidad en educación, un análisis teórico-jurídico, así como la conceptualización de los indicadores para la correlación del IPC y la prueba PISA.

\section{EL DERECHO A LA EDUCACIÓN}

El derecho a la educación es reconocido por los principales instrumentos de $\mathrm{DDHH}$, tanto universales como regionales. Ha sido objeto de tratamiento, directo o indirecto, por todos y cada uno de los tratados internacionales que integran el sistema de tratados de Naciones Unidas y está presente, también, en diversas observaciones generales de Comités de seguimiento de tratados de dicha organización. ${ }^{3}$ Este derecho se integra por gran número de derechos específicos diseminados a lo largo no solo de instrumentos internacionales sino también nacionales.

- Solo participar en un estudio: Somalia, Afganistan, Haití, Venezuela, Paraguay, Angola, Cuba, entre otros más de 100 países fueron considerados en el IPC de TI, pero no participan en la prueba PISA, por lo cual fueron excluidos del estudio al ser imposible correlacionar con una sola medida.

- Diferente conformación política para cada estudio: Corea, que aparece en la prueba PISA como Korea, mientras que en el IPC es considerada como Corea del Sur, China que participa en el IPC como China, mientras que en PISA entontramos: Hong Kong-China, Shanghai-China, Macao-China y Chinese Taipei, por lo cual podría implicar un sesgo al correlacionar sus resultados.

3 Puede revisarse, al respecto: artículo 26 de la Declaración Universal de los Derechos Humanos; artículo 5 de la Convención Internacional sobre la Eliminación de todas las formas de Discriminación Racial; artículo 13 del Pacto Internacional de Derechos Económicos, Sociales y Culturales; artículo 29 de la Convención sobre los Derechos del Niño; artículo 24 de la Convención sobre los Derechos de las Personas con Discapacidad; artículo 5 de la Convención relativa a la lucha contra las Discriminaciones en la Esfera de la Enseñanza; artículo XII de la Declaración Americana de los Derechos y Deberes del Hombre; artículo 3 de la Carta de la Organización de los Estados Americanos; y, artículo 13 del Protocolo adicional a la Convención Americana en materia de Derechos Económicos, Sociales y Culturales. Además, puede revisarse: Oficina del Alto Comisionado de las Naciones Unidas para los Derechos Humanos. (El Sistema de Tratados de Derechos Humanos de Naciones Unidas, 2007).
El Comité de Derechos Económicos Sociales y Culturales (CDESC) sostiene que el derecho a la educación incluye, al menos, el derecho a recibir educación, el derecho a la enseñanza primaria, el derecho a la enseñanza secundaria, el derecho a la enseñanza técnica y profesional, el derecho a la enseñanza superior, el derecho a la educación fundamental y el derecho a la libertad de enseñanza. (Comité de Derechos Económicos, Sociales y Culturales, 1999). La advertencia: "al menos» que hace el CDESC es relevante dado que la derivación de los derechos ínsitos en un derecho humano conlleva la tarea de revisión de todos los instrumentos relevantes al respecto. (Courtis, 2008, p.).

Así, solo a manera de ejemplo, pueden señalarse como derechos específicos del derecho a la educación: la libertad de pensamiento, conciencia y religión en el ámbito de la enseñanza, consagrado en el artículo 18 del Pacto Internacional de Derechos Civiles y Políticos, y el derecho de los hijos de trabajadores migratorios de tener acceso a la educación en igualdad de condiciones de trato con los nacionales del país receptor, reconocido en el artículo 30 de la Convención internacional sobre la protección de los derechos de todos los trabajadores migratorios y de sus familiares.

El derecho a la enseñanza primaria y el derecho a la enseñanza secundaria constituyen derechos específicos del derecho a la educación, los cuales implican una serie de obligaciones estatales que los particularizan frente a otros niveles educativos. Así, aun cuando los derechos a la enseñanza primaria y secundaria suponen algunas obligaciones estatales idénticas con la educación técnica y profesional, la superior y la fundamental, tales como las relativas a que todas ellas deben responder a la orientación de la que se dota a la educación desde los estándares internacionales en materia de $\mathrm{DDHH}$.

Los instrumentos internacionales en materia de derechos humanos, tanto universales como 
regionales, dotan de orientación a la educación que ha de recibirse por parte de los Estados signatarios de éstos. De esta forma, se reconoce que la educación que se reivindica como derecho humano no hace alusión a cualquier tipo de aquella, sino a una de tipo específico, es decir, a una educación que: 1) Tienda al pleno desarrollo de la personalidad humana, del sentido de su dignidad y de su autoestima, tanto individual como de la cultura a la que pertenecen las personas; 2) Tienda al fortalecimiento del respeto de los DDHH y de las libertades fundamentales; 3) Favorezca la comprensión, la tolerancia y amistad entre las naciones y todos los grupos étnicos y religiosos; 4) Promueva el espíritu de igualdad entre los sexos; 5) Capacite a las personas para participar de forma efectiva en una sociedad libre; 6) Coadyuve con el combate y la erradicación de la discriminación; 7) Promueva la paz y el desarrollo, así como las actividades de las Naciones Unidas para el mantenimiento de la paz; e 8) Inculque en los educandos el respeto por el medio ambiente.

Las de que todos los niveles formativos conllevan las obligaciones del Estado de respetar, proteger y garantizar los derechos; ${ }^{4}$ de que la educación

4 Comité de Derechos Económicos, Sociales y Culturales, La aplicación interna del Pacto Internacional de Derechos Económicos, Sociales y Culturales, Observación General Número 9, 1998; SANDOVAL Terán, Areli (Coord.), Los derechos Económicos, Sociales y Culturales: exigibles y justiciables. Preguntas sobre los DESC y el protocolo facultativo del Pacto Internacional de Derechos Económicos, Sociales y Culturales, Espacio DESC-PIDHDD-ONUDH-OXFAM-Novib-Hivos-ICCO-Rosa-Luxemburg-Stiftung-ONU-DH, México, 2010; Directrices de Maastricht sobre Violaciones a los Derechos Económicos, Sociales y Culturales, 1997; SERRANO, Sandra y VÁZQUEZ, Daniel (Coord.), Guía de Estudio de la Materia: seminario sobre derechos civiles. Maestría en Derechos Humanos y Democracia 2010-2012, Flacso, México, 2010; JANA, Andrés, "La eficacia horizontal de los derechos fundamentales" En Roberto Saba, Derechos Fundamentales, Del Puerto, Argentina, 2004; y, MIJANGOS González, Javier, "La doctrina de la Drittwirkung der Grundrechte en la jurisprudencia de la Corte Interamericana de Derechos Humanos", En Derecho Procesal Constitucional. 50 años de desarrollo científico: 1956-2006. Estudios en homenaje a Héctor Fix-Zamudio, Porrúa-Universidad Nacional Autónoma de México, México, 2007; Corte Interamericana de Derechos en todos sus niveles debe satisfacer de forma conjunta los elementos de accesibilidad, disponibilidad, adaptabilidad y aceptabilidad; así como respetar los principios de no discriminación, progresividad y prohibición de regresividad y del máximo uso de los recursos disponibles (Carbonell, M., 2006, p. 49); se particularizan respecto a algunas obligaciones.

En ese orden de ideas, la enseñanza primaria es vista como la que debe satisfacer las necesidades básicas de aprendizaje de todas las personas, esto es, como aquella que debe proveer de las herramientas esenciales para el aprendizaje (lectura y escritura, expresión oral, cálculo, solución de problemas) y los contenidos necesarios (conocimientos teóricos y prácticos, valores y aptitudes) para que las personas puedan sobrevivir, desarrollar sus capacidades, vivir y trabajar con dignidad, participar plenamente en el desarrollo, mejorar la calidad de su vida, tomar decisiones fundamentales y continuar aprendiendo (Declaración Mundial sobre Educación para todos, 1990).

El derecho a la educación primaria tiene tres rasgos característicos: universalidad, obligatoriedad y gratuidad. El primero de ellos, implica que es un nivel educativo al que deben acceder todas las personas, sin discriminación alguna; el segundo, conlleva a que nadie tiene derecho a tratar como optativa la decisión de si los niños deberían tener acceso a la enseñanza primaria, pues todos deberían poder hacerlo; la gratuidad significa que esta no debe implicar menoscabo material o financiero alguno. Ligada a dichos rasgos está la obligación estatal de adoptar un plan detallado que incluya toda medida necesaria para garantizar la realización de todos los elementos esenciales del derecho, dentro

Humanos, Caso Velásquez Rodríguez vs Honduras. Sentencia del 29 de julio de 1988; y, Comité de los Derechos del Niño, La salud y el desarrollo de los adolescentes en el contexto de la Convención sobre los Derechos del Niño, Observación general No. 4, 2003. 
de los cuales se ubica, como ya se ha dicho, la aceptabilidad de la educación, (Comité de Derechos Económicos, Sociales y Culturales, 1999), misma que implica que tanto la forma como el fondo de la educación recibida debe ser aceptable, esto es, adecuada culturalmente, pertinente y de buena calidad; cualidad esta última sobre la que se volverá más tarde.

Lo anterior, dicho de forma breve, implica que el derecho a la educación no se satisface con la simple oferta educativa o con el acceso a la misma, sino que se requiere que esta sea aceptable, es decir, que sea compatible con los $\mathrm{DDHH}$ y, entre otras cosas, de calidad.

El derecho a la enseñanza secundaria es visto como aquel que implica la conclusión de la educación básica, la consolidación del fundamento para el desarrollo humano y para el aprendizaje a lo largo de la vida. Se entiende que la realización efectiva de este prepara a los estudiantes para la enseñanza superior y profesional. A diferencia de la primaria, debe ser generalizada, procurándose en todo momento la progresividad en su gratuidad.

Como puede observarse, los derechos a la enseñanza primaria y a la enseñanza secundaria, constituyen un continuo, la educación básica, en el que el propósito principal es pertrechar a las personas y consolidar en las mismas las herramientas esenciales para el aprendizaje y los contenidos básicos para el desenvolmiento de su propia vida. De ahí que el derecho a la educación, y muy especialmente los derechos educativos en comento, constituyan un medio indispensable para la realización de los demás $\mathrm{DDHH}$; así las cosas, una educación básica que no logra sus objetivos, poco o nada abona al mejoramiento de las condiciones de vida de las personas y de los pueblos.

De lo anterior deriva la obligación jurídica concreta de los Estados de establecer y mantener un sistema para comprobar si la educación se orienta hacia los fines que se le asignan; si cumple con los elementos de accesibilidad, disponibilidad, adaptabilidad y aceptabilidad y con los principios de aplicación que le rigen.

Como se adelantó, lo que interesa es el tema de la calidad educativa, misma que hace parte del elemento de aceptabilidad, pero sobre esto se vuelve en los siguientes apartados. Baste lo dicho para tener un marco general del derecho a la educación y un acercamiento sobre los derechos educativos objeto de estudio de este documento. Adicionalmente, lo anterior permite observar la importancia que tienen las evaluaciones sobre calidad educativa, en la medida en que ellas permiten conocer los avances específicos de los países en torno a la obligación de satisfacer el elemento de calidad en la educación y en el desarrollo de las competencias, en el caso que interesa a este estudio de educación básica.

\section{LA PRUEBA PISA Y LA CALIDAD EDUCATIVA}

Una vez establecido que la educación de calidad hace parte del derecho humano a la educación, que los Estados tienen la obligación de ofrecer una educación de calidad y que los resultados de las evaluaciones son un indicador de calidad en los procesos de enseñanza-aprendizaje; puede entenderse que la evaluación es un proceso paralelo a la educación que ofrece información útil acerca de cómo mejorar el proceso y si éste ha cumplido sus objetivos.

La evaluación se nutre de diferentes instrumentos de medición que permiten tener datos concretos sobre la realidad que va a evaluarse, los cuales al ser interpretados y utilizados como fuentes de información para la toma de decisiones y la mejora del proceso constituyen la evaluación; así, el concepto de evaluación va más allá del resultado de una nota o medida, la medición es una etapa que no debe confundirse con la evaluación, no obstante, para los alcances de este estudio basta con un indicador de medición de la calidad educativa para vincularlo y analizar su relación con los resultados de la percepción de la corrupción. 
Innumerables especialistas como Tamez o Neville concuerdan en que la evaluación educativa es un concepto muy amplio, el cual se puede aplicar a gran cantidad de momentos, procesos o actores educativos, tales como una institución, entidad o escuela, el aprendizaje, los profesores, los métodos, la administración, los estudiantes o el propio sistema educativo en su conjunto.

Estos procesos de evaluación educativa pueden nutrirse de un número aún más amplio de medidas que reflejan indicadores de los sistemas y procesos educativos, por ejemplo: número de profesores, cobertura general, deserción, accesibilidad por género, eficiencia terminal, grado de estudios en la planta académica, tipo de contratación, financiamiento, calificaciones en los estudiantes, número de planteles, entre otros.

A pesar de que los tratados internacionales generalmente se centran más en aspectos cualitativos (UNESCO, 2004), en buena parte, todos estos factores de evaluación y medidas se justifican en la intención de buscar indicadores de calidad educativa, concepto que, como se ha dicho, se vincula con el derecho a la educación puesto que este derecho no sólo implica contar con acceso a un sistema educativo, sino también que el tiempo $y$ recursos que invierte cada estudiante en este proceso refleje una formación de calidad.

En términos del Fondo de Naciones Unidas para la Infancia (UNICEF, 2000)el concepto de calidad en la educación básica es complejo y multifacético, otros conceptos como eficiencia terminal, equidad y cobertura a menudo se usan como sinónimos. No obstante, existe un considerable consenso en torno a las dimensiones esenciales de la calidad de la educación básica hoy en día:

- Ambientes saludables, seguros, protectores y sensibles al género, que proporcionan recursos y servicios adecuados.

- Contenidos que se reflejan en currículos y materiales pertinentes para la adquisición de competencias básicas, especialmente en las áreas de alfabetización, aritmética y competencias prácticas para la vida.

- Procesos mediante los cuales los docentes capacitados utilizan una educación centrada en el estudiante.

- Todo ello permite obtener resultados que abarcan una formación integral de conocimientos, habilidades y actitudes, los cuales están vinculados a objetivos nacionales para la educación y la participación positiva en la sociedad.

De lo anterior puede derivarse que los resultados de desarrollo de competencias en los estudiantes sea uno de los principales indicadores de calidad educativa en los diferentes sistemas nacionales, un sistema que forma integralmente a estudiantes en sus competencias básicas para la vida es un sistema que cumple sus objetivos y por ende cuenta con estándares mínimos de calidad.

Al respecto, la Organización para la Cooperación y el Desarrollo Económico (OCDE) creó en 1997 el Programa para la Evaluación Internacional de Estudiantes el cual es un estudio internacional que tiene como objetivo evaluar sistemas educativos de todo el mundo poniendo a prueba las habilidades y conocimientos de estudiantes de 15 años de edad, hasta la fecha alrededor de 70 países de diferentes regiones han participado en esta prueba que se realiza cada 3 años.

En palabras de la propia OCDE, el programa PISA, «Evalúa la calidad, equidad y eficiencia de los sistemas escolares de unos 70 países que, en su conjunto, componen nueve décimas de la economía mundial. Representa un compromiso de los gobiernos para hacer un seguimiento de los resultados de los sistemas educativos de forma regular dentro de un marco acordado internacionalmente» (OCDE, 2010).

El programa se basa fundamentalmente en las competencias desarrolladas por los estudiantes como principal fuente de información que refleja los resultados que logra el sistema educativo en su conjunto. "Examina el grado en que los estudiantes que están acabando 
la educación obligatoria han adquirido parte de los conocimientos y de las capacidades que necesitan para participar plenamente en una sociedad moderna. Se centra sobre todo en lectura, matemáticas y ciencias» (OCDE, 2010). Dicho en términos de derechos, se trata de una prueba que pretende conocer el grado de cumplimiento de la obligación del desarrollo de competencias elementales de los estudiantes, lo cual hace parte del derecho a la educación básica.

Para la elaboración de la prueba PISA, expertos de los países participantes elaboran un marco base por cada área de evaluación, que posteriormente es aprobado por los gobiernos de cada país, sobre dicho marco se elaboran los instrumentos de evaluación conformados por unidades, el formato de las preguntas es diverso, alrededor del $50 \%$ son preguntas de opción múltiple, mientras que en el otro $50 \%$ se encuentran dicotómicas, de ordenar series y completar enunciados, entre otras. El tiempo total de las pruebas es de 390 minutos. Posterior a su validación las pruebas fueron aplicadas a una muestra estadísticamente determinada de 470000 estudiantes de los diferentes países participantes, por ello los resultados de PISA gozan de una amplia aceptación internacional, además de validez interna, (OCDE, 20109, dichos resultados para la última prueba publicada (2009), se muestran en la tabla I, considerando exclusivamente, como se adelantó, los países que participan en ambas mediciones: PISA e IPC.

Tabla I. Resultados de la Prueba PISA 2009 (OCDE, 2010, p.16)

\begin{tabular}{|c|c|c|c|}
\hline País & Lectura & Matemáticas & Ciencias \\
\hline Finlandia & 536 & 541 & 554 \\
\hline Hong Kong & 533 & 555 & 549 \\
\hline Singapur & 526 & 562 & 542 \\
\hline Canadá & 524 & 527 & 529 \\
\hline Nueva Zelanda & 521 & 519 & 532 \\
\hline Japón & 520 & 529 & 539 \\
\hline Australia & 515 & 514 & 527 \\
\hline Países Bajos & 508 & 526 & 522 \\
\hline Bélgica & 506 & 515 & 507 \\
\hline Noruega & 503 & 498 & 500 \\
\hline Estonia & 501 & 512 & 528 \\
\hline Suiza & 501 & 534 & 517 \\
\hline Polonia & 500 & 495 & 508 \\
\hline Islandia & 500 & 507 & 496 \\
\hline Estados Unidos & 500 & 487 & 502 \\
\hline Suecia & 497 & 494 & 495 \\
\hline Alemania & 497 & 513 & 520 \\
\hline Irlanda & 496 & 487 & 508 \\
\hline Francia & 496 & 497 & 498 \\
\hline
\end{tabular}




\begin{tabular}{|c|c|c|c|}
\hline País & Lectura & Matemáticas & Ciencias \\
\hline Dinamarca & 495 & 503 & 499 \\
\hline Reino Unido & 494 & 492 & 514 \\
\hline Hungría & 494 & 490 & 503 \\
\hline Portugal & 489 & 487 & 493 \\
\hline Macao & 487 & 525 & 511 \\
\hline Italia & 486 & 483 & 489 \\
\hline Eslovenia & 483 & 501 & 512 \\
\hline Grecia & 483 & 466 & 470 \\
\hline España & 481 & 483 & 488 \\
\hline República Checa & 478 & 493 & 500 \\
\hline Eslovaquia & 477 & 497 & 490 \\
\hline Croacia & 476 & 460 & 486 \\
\hline Israel & 474 & 447 & 455 \\
\hline Luxemburgo & 472 & 489 & 484 \\
\hline Austria & 470 & 496 & 494 \\
\hline Lituania & 468 & 477 & 491 \\
\hline Turquía & 464 & 445 & 454 \\
\hline Emiratos Árabes Unidos & 459 & 453 & 466 \\
\hline Rusia & 459 & 468 & 478 \\
\hline Chile & 449 & 421 & 447 \\
\hline Serbia & 442 & 442 & 443 \\
\hline Bulgaria & 429 & 428 & 439 \\
\hline Uruguay & 426 & 427 & 427 \\
\hline México & 425 & 419 & 416 \\
\hline Rumania & 424 & 427 & 428 \\
\hline Tailandia & 421 & 419 & 425 \\
\hline Trinidad y Tobago & 416 & 414 & 410 \\
\hline Bolombia & 413 & 381 & 402 \\
\hline Bontenegro & 412 & 386 & 405 \\
\hline Jordania & 408 & 403 & 401 \\
\hline Túnez & 405 & 387 & 415 \\
\hline Indonesia & 404 & 371 & 401 \\
\hline Kazajstán & 302 & 371 & 383 \\
\hline & 398 & 388 & 401 \\
\hline & 405 & 400 \\
\hline
\end{tabular}




\begin{tabular}{|c|c|c|c|}
\hline País & Lectura & Matemáticas & Ciencias \\
\hline Albania & 385 & 377 & 391 \\
\hline Qatar & 372 & 368 & 379 \\
\hline Panamá & 371 & 360 & 376 \\
\hline Perú & 370 & 365 & 369 \\
\hline Azerbaiyán & 362 & 431 & 373 \\
\hline Kirguisitán & 314 & 331 & 330 \\
\hline
\end{tabular}

Como se puede apreciar, existen marcadas diferencias entre los participantes, Finlandia, Hong Kong y Singapur son los países que encabezan la lista, mientras que Chile es el primero de Latinoamérica; en la mayoría de los países el desarrollo de competencias se encuentra en un nivel bajo respecto del promedio de la OCDE, ${ }^{5}$ esta medida nos permitirá a continuación correlacionar los resultados educativos de PISA con el IPC de TI.

\section{CORRUPCIÓN Y VULNERACIÓN DE DERECHOS HUMANOS}

El argumento de la corrupción como medio de vulneración de derechos humanos o, más genéricamente, de la relación entre corrupción y derechos humanos (DDHH), fue avanzada a finales de la década de los años noventa del siglo pasado por autores como Rajagopal, Kofele-Kale y Kumar (Posadas, 2006, p.27).

El primero de ellos trazó la dialéctica entre corrupción, legitimidad y DDHH, planteando que estos y la lucha contra la corrupción constituían dos caras de una misma moneda: la legitimidad del Estado. Analiza en qué coinciden, en qué se diferencian y cuáles son los espacios de complementación entre los discursos de los DDHH y la lucha contra la

5 Promedios de la OCDE: Lectura 493, Matemáticas 496, Ciencias 501. corrupción (Rajagopal, 1999, pp. 495-506). Por su parte, Kofele-Kale y Kumar son vistos como los principales proponentes del reconocimiento de un derecho humano a vivir en un mundo sin corrupción (Gruenberg, 2007, pp. 12-13); desde su perspectiva, la importancia de tal reconocimiento reside en que éste supondría la creación y aprovechamiento de mecanismos para la promoción y protección de este derecho, protección que encuentra su razón de ser en que la corrupción atenta contra los valores fundamentales de la dignidad humana y la igualdad política de las personas; ellos adoptan una perspectiva internacional, pues consideran que a partir de ella podría elevarse la corrupción a la condición de crimen internacional y se facilitaría con ello su integración en el derecho interno de los Estados (Kumar, 2002).

Hoy día es claro que la relación entre corrupción y $\mathrm{DDHH}$ ha sido aceptada como relevante tanto por la doctrina de los DDHH como por la de combate a la corrupción, así como por diversos organismos internacionales.

En efecto, son diversos los organismos que han solicitado o que han realizado estudios respecto a la relación entre corrupción y DDHH. Así, el Programa de las Naciones Unidas para el Desarrollo publicó un estudio en el que se concluyó que la corrupción tiene un impacto desproporcionado sobre los pobres, dado que esta distorsiona las decisiones públicas a favor de ricos y poderosos y reduce la capacidad del Estado para proporcionar seguridad social; 
asimismo, se afirmó que el impacto negativo de la corrupción sobre el desarrollo humano y los DDHH ya no está en tela de juicio (Pilapitiya, 2004, pp. 9-10).

La ONU creó en el año 2002 la Relatoría Especial sobre las repercusiones de la corrupción en la realización y disfrute de los $\mathrm{DDHH}$, en particular sobre los derechos económicos, sociales y culturales (DESC), a través de la cual se ha reconocido que la corrupción puede generar importantes menoscabos a los derechos civiles y políticos, y a los económicos, sociales y culturales; por su parte, TI ha sostenido que la corrupción es la causa y el problema esencial de muchas violaciones de DDHH (Transparencia Internacional, 2008, p.1), y el Consejo Internacional para el estudio de los Derechos Humanos publicó un estudio en el que se afirma que, cotidiana y silenciosamente, la corrupción viola DDHH de las personas de forma sistemática (Gruenberg, 2010, p.1). En suma, el ambiente que prevale entorno a la relación entre corrupción y DDHH apunta a que estudios y análisis observan un impacto negativo de aquella en estos y a la necesidad de generar una estrategia de vinculación entre los discursos y prácticas del movimiento de DDHH y anticorrupción.

Sin embargo, cuando se trata de establecer el vínculo entre corrupción y violación de $\mathrm{DDHH}$ la literatura se divide. Así, autores como Goodwin y Rose ( 2010) sostienen que este tipo de vinculación resulta estéril porque la agenda contra la corrupción descansa en la ideología del neoliberalismo, misma que pretende ver a la corrupción como un factor explicativo mono-causal de los fracasos del desarrollo y que pretende poner de relieve unos aspectos y ocultar otros. En este sentido, para ellos, el intento de vincular los $\mathrm{DDHH}$ con la corrupción es parte de una tendencia más amplia en la que las acusaciones de corrupción juegan un papel de legitimación de las políticas adoptadas por los organismos internacionales. Goodwin y Rose admiten que la corrupción tiene un impacto negativo sobre la realización de todos los $\mathrm{DDHH}$, pero niegan que el vínculo pueda plantearse desde los postulados reinantes en el combate a la corrupción. Desde su perspectiva, el vínculo entre corrupción y DDHH solo podrá ser potenciado si se parte de la idea de que la realización de los DDHH afecta la existencia de la corrupción (Goodwin \& Rose, 2010, pp. 11-15).

Bryane y Habit (2010, pp. 1-8) señalan que la relación directa entre corrupción y $\mathrm{DDHH}$ propuesta por algunos autores está lejos de ser clara. Así, afirman que la corrupción lleva a violaciones de $\mathrm{DDHH}$, pero no (de inmediato y para efectos de litigio) los causan. Para sustentar sus argumentos utilizan un caso hipotético y a través de él muestran que la corrupción actúa como un factor contribuyente para la vulneración de $\mathrm{DDHH}$, pero no puede pensarse como la causa de la misma. Para ellos, el fenómeno de la corrupción es mucho más complejo; de ahí que no deba plantearse de manera directa un vínculo entre ella y la violación de DDHH. Con base en tales ideas, los autores proponen clasificar, desde una perspectiva práctica-litigiosa, los efectos de la corrupción sobre los $\mathrm{DDHH}$ ya sea que estos precedan, ocurran durante o después de la vulneración de un derecho.

Por otra parte, Thuo (2009, pp. 125-148) muestra cómo los derechos individuales y procesales han sido utilizados en Kenia para derrotar investigaciones y procesamientos por corrupción de altos funcionarios gubernamentales y cómo algunas reformas anticorrupción pueden ser incompatibles con los DESC de pobres $y$ marginados. De ahí que para él la relación entre DDHH y corrupción sea ambigua, pues mientras la corrupción afecta negativamente la protección de los DDHH, estos pueden ayudar a la corrupción a florecer.

Bacio-Terracino (2008, pp.1-8) proporciona un esquema analítico para determinar cuándo las prácticas corruptas vulneran $\mathrm{DDHH}$, mismo que ha sido adoptado en su integralidad por el Consejo Internacional para las Políticas de los Derechos Humanos y por TI. En esta perspectiva, se piensa que para aplicar el marco conceptual de $\mathrm{DDHH}$, con potencial 
efecto legal, es necesario distinguir entre: (1) prácticas corruptas que violan directamente un derecho humano, (2) prácticas corruptas que conducen a la violación de un derecho humano (pero que, en sí mismas, no violan un derecho), y (3) prácticas corruptas en las que no puede establecerse vínculo causal con una violación de DDHH específicos. De ahí que pueda diferenciarse entre violaciones a los DDHH por corrupción directas, indirectas y remotas. Ya sea que el acto corrupto se utilice de forma deliberada como medio para la violación de un derecho (directa); que la corrupción sea un factor esencial que contribuye en una cadena de acontecimientos que, eventualmente, conducen a la violación de un derecho (indirecta); o bien, que la corrupción solo sea un factor de entre muchos que entran en juego y que esta solo cumpla un papel de contribución en la vulneración.

Es importante destacar que estudios empíricos han demostrado que la corrupción tiende a limitar la inversión gubernamental en áreas que no resultan atractivas para funcionarios corruptos, entre los cuales destaca la educación y que la corrupción afecta a los sectores más desprotegidos y de ingresos más bajos, toda vez que estos son los mayores beneficiarios de servicios de salud y educación públicos (Posadas, 2006, p. 22).

En adición a lo anterior, cabe decir que desde la perspectiva teórica también se han dado acercamientos al tema corrupción y educación; sin embargo, no se conocen perspectivas empíricas como la que aquí se presenta a partir de un estudio estadístico; de igual forma, se carece de un consenso respecto a qué tipo de impacto puede estar teniendo la corrupción en la educación y en relación a qué aspectos de la misma, de ahí que este estudio pueda proporcionar un acercamiento a una pequeña parcela de la enorme complejidad que plantea la relación abordada.

\section{BUSCANDO BAJO LAS PIEDRAS... ¿CÓMO CUANTIFICAR LA CORRUPCIÓN? EL ÍNDICE DE PERCEPCIÓN DE TRANSPARENCIA INTERNACIONAL (TI) Y SU CONFIABILIDAD}

La corrupción constituye un mal secreto y silencioso que afecta a las diferentes sociedades. Pese a lo evidente que pueda ser resulta muy complicada su medición, dado que en muchas ocasiones implica actos en los cuales los involucrados se han beneficiado indebidamente $y$, en consecuencia, su intención no será de denuncia sino de secrecía respecto del acto.

Por ello, la tarea de cuantificar u obtener datos exactos sobre la corrupción en un país se asemeja a la labor de buscar bajo las piedras algún espécimen que tan pronto se ve descubierto busca de inmediato un nuevo escondite sin dejar pista atrás de su paradero.

«A diferencia de otras faltas, como el robo y el asalto, la corrupción, por lo general, carece de una víctima inmediata que tenga interés en elaborar un informe y proporcionar evidencia. (...) Peor aún, varias de las funciones desempeñadas por la función pública pueden ser usadas para ocultar la corrupción y sus efectos a menos que dichas funciones sean a su vez cuidadosamente supervisadas». (Johnston, 2005). 
En este sentido, otro riesgo al medir la corrupción es caer en situaciones paradójicas, como la que se presenta cuando países con alto índice de corrupción pueden ser aquellos donde se registra el menor número de sanciones o expedientes con relación al tema, ya que a mayor corrupción en la esfera pública de un país menores serán las posibilidades de denuncias exitosas y la expectativa de éxito en las mismas.

Es por ello que diferentes indicadores para medir la corrupción se han constituido con base en la percepción de las personas, lo cual, si bien tiene un sesgo subjetivo, sin duda arroja una visión más cercana a la realidad que la que se podría obtener al analizar estadísticas de denuncia $u$ algún otro dato concreto sobre procesos judiciales de corrupción. Al construir su percepción y manifestar una sencilla opinión sobre este problema, las personas pueden reflejar con mayor honestidad su experiencia.

Entre dichos indicadores, el IPC publicado por TI ha sido uno de los más aceptados y difundidos a nivel mundial, « el cual ha aparecido anualmente desde 1995 y podría ser descrito como un índice de índices. Es decir, el IPC se presenta como un promedio de varias encuestas, las cuales varían de un mínimo de tres hasta más de una docena» (Johnston, 2005, p. 360).

En efecto, el IPC se constituye con base en datos recopilados de diversas fuentes, en lo particular para el índice 2009 se incluyeron estudios de 2008 y 2009, combinando evaluaciones de informantes clave con los proporcionados por las siguientes fuentes de información: Banco Africano de Desarrollo, Banco Asiático de Desarrollo, Bertelsmann Foundation, Economist Intelligence Unit, Freedom House, Global Insight y el Banco Mundial, cada uno de las cuales tiene estudios que miden el alcance general de la corrupción (frecuencia y/o magnitud de los sobornos) en el sector público y político y ofrecen una clasificación de países. Posteriormente, las puntuaciones se someten a una transformación beta ${ }^{6}$ para ser equiparables y se promedian para obtener una misma medida aplicable a todos los países, la cual, de acuerdo a TI, (2009) arroja resultados con un intervalo de confianza del $90 \%{ }^{7}$

Por lo anterior, pese a la existencia de algunas limitaciones que en el mismo estudio se reconocen, «el IPC ha sido extensamente usado por los investigadores; también ha sido propuesto y discutido como una pauta para encausar la ayuda internacional y las reformas internas de los países» (Johnston, 2005, p.360), en este sentido, se considera la medida idónea sobre corrupción requerida para este estudio correlacional.

Conviene mencionar que el IPC es una medida que se integra con valores de 0 a 10 , en la cual 0 representa el máximo nivel de corrupción, mientras que las medidas más cercanas a 10 son calificaciones positivas de baja corrupción. TI considera que una puntuación inferior a 5 implica una percepción de alta corrupción. En la tabla II, se presenta esta medida, junto con el lugar mundial que ocupó cada país considerado para este estudio, dentro de una lista de 180 países en total; asimismo la desviación estándar y el intervalo de confianza en las encuestas utilizadas de origen para calcular el IPC, lo cual nos habla de la estabilidad estadística que buscan los resultados. ${ }^{8}$

$6 \quad$ Esta transformación permite que todos los datos de las diferentes encuestas e índices, puedan interpretarse en una escala de 1 a 10, independientemente de la escala en la que hayan sido formulados en su origen.

7 Este intervalo indica la probabilidad de que el valor verdadero del parámetro se encuentre en el valor estimado. (Bosch, 2005, p. 397).

8 Para conocer con detalle un conjunto de datos, no basta con conocer las medidas de tendencia central en este caso la media o promedio que indica el IPC, otros datos como la desviación estándar refieren el grado de dispersión de los datos con respecto al valor promedio (Bosch, 2005). 
Tabla II. Índice de Percepción de la Corrupción 2009.(Transparencia Internacional, 2009)

\begin{tabular}{|c|c|c|c|c|c|c|}
\hline \multirow[t]{2}{*}{$\begin{array}{c}\text { Lugar } \\
\text { mundial }\end{array}$} & \multirow[t]{2}{*}{ País } & \multirow[t]{2}{*}{ IPC } & \multirow[t]{2}{*}{$\begin{array}{l}\text { Desviación } \\
\text { estándar }\end{array}$} & \multirow[t]{2}{*}{$\begin{array}{l}\text { Número de } \\
\text { encuestas } \\
\text { utilizadas }\end{array}$} & \multicolumn{2}{|c|}{$\begin{array}{l}\text { Intervalo de } \\
\text { confianza }\end{array}$} \\
\hline & & & & & Mínimo & Máximo \\
\hline 1 & Nueva Zelanda & 9.4 & 0.3 & 6 & 9.1 & 9.5 \\
\hline 2 & Dinamarca & 9.3 & 0.3 & 6 & 9.1 & 9.5 \\
\hline 3 & Singapur & 9.2 & 0.3 & 9 & 9 & 9.4 \\
\hline 3 & Suecia & 9.2 & 0.2 & 6 & 9 & 9.3 \\
\hline 5 & Suiza & 9 & 0.1 & 6 & 8.9 & 9.1 \\
\hline 6 & Finlandia & 8.9 & 0.8 & 6 & 8.4 & 9.4 \\
\hline 6 & Países Bajos & 8.9 & 0.2 & 6 & 8.7 & 9 \\
\hline 8 & Canadá & 8.7 & 0.3 & 6 & 8.5 & 9 \\
\hline 8 & Australia & 8.7 & 0.6 & 8 & 8.3 & 9 \\
\hline 8 & Islandia & 8.7 & 1.3 & 4 & 7.5 & 9.4 \\
\hline 11 & Noruega & 8.6 & 0.7 & 6 & 8.2 & 9.1 \\
\hline 12 & Hong Kong & 8.2 & 0.5 & 8 & 7.9 & 8.5 \\
\hline 12 & Luxemburgo & 8.2 & 0.9 & 6 & 7.6 & 8.8 \\
\hline 14 & Alemania & 8 & 0.5 & 6 & 7.7 & 8.3 \\
\hline 14 & Irlanda & 8 & 0.5 & 6 & 7.8 & 8.4 \\
\hline 16 & Austria & 7.9 & 0.7 & 6 & 7.4 & 8.3 \\
\hline 17 & Japón & 7.7 & 0.5 & 8 & 7.4 & 8 \\
\hline 17 & Reino Unido & 7.7 & 0.7 & 6 & 7.3 & 8.2 \\
\hline 19 & Estados Unidos & 7.5 & 0.9 & 8 & 6.9 & 8 \\
\hline 21 & Bélgica & 7.1 & 0.4 & 6 & 6.9 & 7.3 \\
\hline 22 & Qatar & 7 & 1.8 & 6 & 5.8 & 8.1 \\
\hline 24 & Francia & 6.9 & 0.6 & 6 & 6.5 & 7.3 \\
\hline 25 & Chile & 6.7 & 0.4 & 7 & 6.5 & 6.9 \\
\hline 25 & Uruguay & 6.7 & 0.5 & 5 & 6.4 & 7.1 \\
\hline 27 & Estonia & 6.6 & 0.7 & 8 & 6.1 & 6.9 \\
\hline 27 & Eslovenia & 6.6 & 0.6 & 8 & 6.3 & 6.9 \\
\hline 30 & $\begin{array}{c}\text { Emiratos Árabes } \\
\text { Unidos }\end{array}$ & 6.5 & 1.4 & 5 & 5.5 & 7.5 \\
\hline 32 & España & 6.1 & 0.8 & 6 & 5.5 & 6.6 \\
\hline 32 & Israel & 6.1 & 1 & 6 & 5.4 & 6.7 \\
\hline 35 & Portugal & 5.8 & 0.5 & 6 & 5.5 & 6.2 \\
\hline 43 & Macao & 5.3 & 1.8 & 3 & 3.3 & 6.9 \\
\hline
\end{tabular}




\begin{tabular}{|c|c|c|c|c|c|c|}
\hline \multirow[t]{2}{*}{$\begin{array}{c}\text { Lugar } \\
\text { mundial }\end{array}$} & \multirow[t]{2}{*}{ País } & \multirow[t]{2}{*}{ IPC } & \multirow[t]{2}{*}{$\begin{array}{l}\text { Desviación } \\
\text { estándar }\end{array}$} & \multirow[t]{2}{*}{$\begin{array}{l}\text { Número de } \\
\text { encuestas } \\
\text { utilizadas }\end{array}$} & \multicolumn{2}{|c|}{$\begin{array}{l}\text { Intervalo de } \\
\text { confianza }\end{array}$} \\
\hline & & & & & Mínimo & Máximo \\
\hline 46 & Hungría & 5.1 & 0.9 & 8 & 4.6 & 5.7 \\
\hline 49 & Polonia & 5 & 0.9 & 8 & 4.5 & 5.5 \\
\hline 49 & Jordania & 5 & 1.8 & 7 & 3.9 & 6.1 \\
\hline 52 & República Checa & 4.9 & 1.2 & 8 & 4.3 & 5.6 \\
\hline 52 & Lituania & 4.9 & 0.9 & 8 & 4.4 & 5.4 \\
\hline 56 & Eslovaquia & 4.5 & 0.8 & 8 & 4.1 & 4.9 \\
\hline 61 & Turquía & 4.4 & 0.8 & 7 & 3.9 & 4.9 \\
\hline 63 & Italia & 4.3 & 0.9 & 6 & 3.8 & 4.9 \\
\hline 65 & Túnez & 4.2 & 1.9 & 6 & 3 & 5.5 \\
\hline 66 & Croacia & 4.1 & 0.8 & 8 & 3.7 & 4.5 \\
\hline 69 & Montenegro & 3.9 & 0.6 & 5 & 3.5 & 4.4 \\
\hline 71 & Grecia & 3.8 & 0.8 & 6 & 3.2 & 4.3 \\
\hline 71 & Bulgaria & 3.8 & 1.1 & 8 & 3.2 & 4.5 \\
\hline 71 & Rumania & 3.8 & 1 & 8 & 3.2 & 4.3 \\
\hline 75 & Colombia & 3.7 & 1 & 7 & 3.1 & 4.3 \\
\hline 75 & Brasil & 3.7 & 0.8 & 7 & 3.3 & 4.3 \\
\hline 75 & Perú & 3.7 & 0.7 & 7 & 3.4 & 4.1 \\
\hline 79 & Trinidad y Tobago & 3.6 & 0.8 & 4 & 3 & 4.3 \\
\hline 83 & Serbia & 3.5 & 0.5 & 6 & 3.3 & 3.9 \\
\hline 84 & Tailandia & 3.4 & 0.8 & 9 & 3 & 3.8 \\
\hline 84 & Panamá & 3.4 & 0.4 & 5 & 3.1 & 3.7 \\
\hline 89 & México & 3.3 & 0.3 & 7 & 3.2 & 3.5 \\
\hline 95 & Albania & 3.2 & 0.3 & 6 & 3 & 3.3 \\
\hline 106 & Argentina & 2.9 & 0.4 & 7 & 2.6 & 3.1 \\
\hline 111 & Indonesia & 2.8 & 0.7 & 9 & 2.4 & 3.2 \\
\hline 120 & Kazajstán & 2.7 & 1 & 7 & 2.1 & 3.3 \\
\hline 143 & Azerbaiyán & 2.3 & 0.5 & 7 & 2 & 2.6 \\
\hline 146 & Rusia & 2.2 & 0.4 & 8 & 1.9 & 2.4 \\
\hline 162 & Kirguistán & 1.9 & 0.2 & 7 & 1.8 & 2.1 \\
\hline
\end{tabular}

De los 65 países seleccionados, solo 21 obtuvieron puntuaciones altas por encima de 7, entre los que se destacan Nueva Zelanda, Dinamarca, Singapur, Suecia y Suiza. Sin embargo, conforme la valoración de TI (2009) en lo general, los resultados no son muy favorables, ya que la mayoría de países se ubica por debajo de 5, lo cual muestra que la corrupción continúa acechando en todas las regiones del mundo. 


\section{NOTA SOBRE EL COEFICIENTE DE CORRELACIÓN}

El coeficiente de correlación de Pearson, también conocido como correlación producto momento de Pearson, es una medida de relación lineal entre dos variables continuas. Los valores que adquiere esta correlación se encuentran entre -1 y +1 . Por lo que, si tenemos una correlación +1 significa que existe una relación lineal directa perfecta (positiva), es decir, las puntuaciones bajas de la variable (X) se asocian con las puntuaciones bajas de la variable $(\mathrm{Y})$, mientras que las puntuaciones altas de $\mathrm{X}$ se asocian con los valores altos de Y. Una correlación -1 significa que existe una relación lineal perfecta (negativa) entre las dos variables. Puntuaciones bajas en $\mathrm{X}$ se asocian con los valores altos de $\mathrm{Y}$, mientras las puntuaciones altas en $\mathrm{X}$ se asocian con los valores bajos en Y. En el caso de una correlación 0 se interpreta como la inexistencia de una relación lineal entre las dos variables estudiadas (Elorza, 2000). Castejón aporta la siguiente tabla, en la cual se especifican posibles resultados del índice de correlación y su significado (Castejón, 2011, p. 96).

Tabla III: Interpretación de resultados de la correlación

\begin{tabular}{|c|l|}
\hline $\begin{array}{c}\text { Valor de la } \\
\text { correlación }\end{array}$ & \multicolumn{1}{|c|}{ Significado } \\
\hline-1 & Correlación negativa grande y perfecta \\
\hline$-0,9$ a $-0,99$ & Correlación negativa muy alta \\
\hline$-0,7$ a $-0,89$ & Correlación negativa alta \\
\hline$-0,4$ a $-0,69$ & Correlación negativa moderada \\
\hline$-0,2$ a $-0,39$ & Correlación negativa baja \\
\hline$-0,01$ a $-0,19$ & Correlación negativa muy baja \\
\hline 0 & Correlación nula \\
\hline 0,01 a 0,19 & Correlación positiva muy baja \\
\hline 0,2 a 0,39 & Correlación positiva baja \\
\hline 0,4 a 0,69 & Correlación positiva moderada \\
\hline 0,7 a 0,89 & Correlación positiva alta \\
\hline 0,9 a 0,99 & Correlación positiva muy alta \\
\hline 1 & Correlación positiva grande y perfecta \\
\hline
\end{tabular}

Tal como mencionan Hopkings, Hopkings y Glass: «Las variables que son correlacionadas pueden ser dos variables cuantitativas cualesquiera, como aprovechamiento en historia $y$ aprovechamiento en lectura, velocidad en la carrera corta de las 100 yardas y habilidad para tocar el violín o conservadurismo político y edad» (Hopkings K., \& Hopkings, B., 1997, p.88). Por ello, este coeficiente resultó ideal para la presente investigación, donde se busca conocer la relación entre corrupción y educación.

Para el cálculo del coeficiente de correlación, se utilizan dos variables aleatorias, " $x » y\langle y »$, sobre una misma población estadística, el coeficiente de Pearson, simbolizado como, se calcula con la siguiente fórmula:

$$
P x, y=\frac{\text { oxy }}{\text { oxơy }}=\frac{E[(X-\mu \mathrm{x})(\mathrm{Y}-\mu \mathrm{y})]}{\text { oxơy }}
$$

Donde:

- es la covarianza de (X,Y)

- es la desviación típica de la variable X

- es la desviación típica de la variable Y

Para calcular el coeficiente en esta investigación, se utilizó el software Statistical Package for the Social Sciences, del cual se obtuvo el cálculo de las medidas descritas en el siguiente apartado; asimismo, se probó la significancia ${ }^{9}$ de la correlación utilizando el mismo software.

9 Una forma de probar estadísticamente la significancia de una relación lineal entre $X$ y $Y$, es mediante una prueba de hipótesis como la siguiente:

$\mathrm{H}_{0}: r=0$ El coeficiente de correlación obtenido procede de una población cuya correlación es cero $(p=0)$.

$\mathrm{H}_{a}: r \neq 0$ El coeficiente de correlación obtenido procede de una población cuyo coeficiente de correlación es distinto de cero $(p \neq 0)$.

$\mathrm{Si}$ suponemos que de dos poblaciones normales se extraen muestras de $\mathrm{n}$ pares de datos $(x, y)$, entonces podemos probar la prueba de hipótesis nula, que se supone cierta, se puede demostrar que la distribución muestral del estadístico

$$
t=r \frac{\sqrt{\mathrm{n}-2}}{\sqrt{1-\mathrm{r}^{2}}}
$$




\section{RESULTADOS DE CORRELACIÓN IPC-PISA}

La tabla IV, que se muestra a continuación, contiene los resultados de correlación estadística que se presentan al aplicar el coeficiente de correlación de Pearson a los resultados IPC y PISA que se mostraron previamente en las tablas I y II. ${ }^{10}$

Tiene una distribución $\mathrm{t}$-Student con n-2 g.l. y un nivel de significancia $\alpha$, este se compara con un valor obtenido de las tablas $t_{(a, n-2)}$

Decisión, si $t>t_{(a, n-2)}$ Se rechaza la Hipótesis nula, otra forma de decisión es utilizando el sig, que nos ofrece el software SPSS 19 y lo comparamos con el nivel de significancia a. Sí sig $<0.05$ se rechaza $\mathrm{H}_{0}$. (Murray \& Spiegel, 2002, pp. 314363).

10 La correlación también permite la comprobación de hipótesis:

H0: $r=0$ El coeficiente de correlación obtenido procede de una población cuya correlación es cero $(p=0)$.

Ha: $r \neq 0$ El coeficiente de correlación obtenido procede de una población cuyo coeficiente de correlación es distinto de cero $(p \neq 0)$.

Un $\alpha=0.05$

Un nivel de confianza del $95 \%$

Obtenemos los coeficientes de correlación de Pearson en la tabla 5 y observamos que los valores Sig. Para cada una de las correlaciones es menor a $\mathrm{a}=0.05$, por lo tanto se rechaza la hipótesis nula, los coeficientes de correlación de Pearson provienen de una población cuyo coeficiente es distinto de cero.

Tabla IV, Correlación IPC-PISA

\begin{tabular}{|c|c|c|c|c|}
\hline & IPC & Lectura & Matemáticas & Ciencias \\
\hline IPC Correlación de Pearson & 1 & $.764^{* *}$ & $.749^{* *}$ & $.761^{* *}$ \\
Sig. (bilateral) & & .000 & .000 & .000 \\
\hline
\end{tabular}

En la tabla IV se manifiesta que la correlación entre el IPC y las asignaturas de lectura, matemáticas y ciencias tienen una correlación positiva alta con $0.764,0.749$ y 0.761 respectivamente; del mismo modo, a mayor puntuación en el IPC, mayor será la puntuación de las asignaturas; recordemos que para este caso cuando el IPC aumenta se refiera a una baja corrupción. Por otra parte, podríamos decir que la correlación entre las asignaturas presenta una correlación positiva muy alta, eso quiere decir que si las puntuaciones son altas para una asignatura, por ejemplo la lectura, la puntuación de las otras tiende a ser alta también; por ello, se podría considerar que el IPC tiene un impacto en las tres asignaturas no solo en una.
Para probar esta idea se realizó la prueba Kruskal Wallis $^{11}$ para verificar si existen diferencias

11 La prueba $\mathrm{H}$ de Kruskal-Wallis se aplica para detectar diferencias en las medias entre más de dos poblaciones, basándose en un muestreo aleatorio independiente. La hipótesis se formuló de la siguiente manera.

1) $\mathrm{H}_{0}$ : Las $\mathrm{K}$ poblaciones son idénticas

2) $\mathrm{H}_{\mathrm{a}}$ : Por lo menos dos de las $\mathrm{K}$ poblaciones no son idénticas

3) Fijar nivel de significancia a

4) Región crítica de con K-1 g.l.

5) Estadístico de prueba

$$
H=\frac{12}{n(n+1)} \sum_{i=1}^{k} \frac{T^{2}}{n_{1}}-3(n+1)
$$

6) Decisión: Se rechaza $\mathrm{H}_{0}$ si $\mathrm{H}>$

7) Para el caso del nivel de significancia obtenido en el software SPSS 19, 
significativas en las medias de las asignaturas, procedimiento que permitió comprobar que la percepción de la corrupción no solo impacta en una disciplina sino en todas.

De la misma manera, el coeficiente de determinación ${ }^{12}$ permite comprobar que la correlación es alta, ya que hasta un $58 \%$ de las variaciones en las diferentes asignaturas de la prueba PISA se relacionan con variaciones en los resultados del IPC, como se muestra en la tabla V.

Tabla V. Coeficiente de determinación IPC-PISA.

\begin{tabular}{|l|r|r|}
\hline & $\mathrm{R}$ & \multicolumn{2}{|l|}{$\mathrm{R}^{2}$} \\
\hline IPC \& Lectura & 0.76 & 0.58 \\
\hline IPC \&Matemáticas & 0.75 & 0.56 \\
\hline IPC\& Ciencias & 0.76 & 0.58 \\
\hline
\end{tabular}

Asimismo, haciendo un estudio particular, también puede notarse que varios de los países ubicados en los primeros lugares en el IPC como Finlandia o Nueva Zelanda obtuvieron también

sí sig $<0.05$ se rechaza $\mathrm{H}_{0}$.

Para el caso de las medias la prueba de hipótesis a probar es la siguiente:

$\mathrm{H}_{0}$ : La media de las asignaturas lectura, matemáticas y ciencias es la misma para las tres asignaturas.

$\mathrm{H}$ : Al menos dos medias de las asignaturas son diferentes.

Tabla 7 : Valor sig de Kruskal Wallis

Estadísticos de contrastea,b

\begin{tabular}{|c|c|}
\hline & VAR00003 \\
\hline Chi-cuadrado & .942 \\
Gl & 2 \\
Sig. asintót. & .624 \\
\hline
\end{tabular}

Con un nivel de confianza del $95 \%$ y un nivel de significancia $a=0.05$, obtenemos un sig $=0.624$, Por lo que se observa que sig $=0.62>a=0.05$, por lo tanto no se rechaza $\mathrm{H}_{0}$, es decir no existen diferencias en las medias de las tres asignaturas, con esto podemos decir que la percepción de la corrupción no solo impacta en una disciplina si no que en todas. (Freund, J. \& Simon, G., 1994, pp. 420-479).

12 El coeficiente de determinación expresa que tanto por ciento de las variaciones en $\mathrm{Y}$ son explicadas por X, Tomamos a el IPC como X y a las asignaturas de PISA como Y. los primeros lugares en la prueba PISA; de la misma manera, naciones con alta percepción de corrupción y muy baja calificación en IPC obtuvieron coincidentemente algunos de los últimos lugares en la prueba PISA, ejemplo de Azerbaiyán o Kirguistán.

No obstante que se presentan excepciones co mo el caso de Polonia, país que pese a contar con un IPC de 5 (alta percepción de corrupción) obtuvo buenos resultados en materia educativa con 501 puntos promedio, los mismos que alcanzó Islandia, país que obtuvo 8.7 en IPC (percepción de baja corrupción). Sin embargo, se trata de un caso excepcional, ya que en términos generales la correlación estadística es contundente para aceptar la hipótesis planteada, esto es, a menor percepción de la corrupción en un país corresponden mejores resultados en la evaluación de su calidad educativa.

\section{CONCLUSIÓN}

La educación básica se considera un DDHH, ya que permite el desarrollo de competencias que hoy en día resultan indispensables para desenvolverse en la sociedad y obtener los recursos elementales para la subsistencia; se relaciona con la consecución de otros derechos como la vida digna, trabajo, acceso a la cultura, información, entre muchos otros, por ello resulta fundamental que los diversos países proporcionen una educación básica de calidad. En la tarea de obtener mediciones de carácter cuantitativo que resulten ilustrativas de los temas de corrupción y calidad educativa en diferentes países, los índices IPC de TI y PISA de la OCDE, resultan dos parámetros suficientemente acreditados, tanto por su prestigio internacional como por sus criterios internos de validez, pese a diversos aspectos perfectibles en ambas pruebas.

Tomando en consideración lo anterior, se concluye que las percepciones de alta corrupción en el IPC tienen una correlación estadística superior al 0.75 , con bajos resultados en el desarrollo 
de competencias de los estudiantes en la prueba PISA. Esta correlación es alta y consistente para las diferentes áreas de formación educativa, sin presentar una variación significativa para el caso de habilidades lectoras, matemáticas o en ciencias.

Lo anterior permite afirmar que, en términos generales, los países en los que existe baja percepción de corrupción en sus esferas pública y privada, tienden a desarrollar también sistemas educativos que resultan mejor evaluados en calidad, en específico en cuanto al desarrollo de las competencias básicas en sus estudiantes. Lo cual permite vislumbrar la forma en la que la corrupción puede impactar el derecho a una educación de calidad.

\section{REFERENCIAS}

Abramovich, C. (1997). Hacia la exigibilidad de los derechos económicos, sociales y culturales. Estándares internacionales y criterios de aplicación ante los tribunales locales. En Abregú, M., \& Courtis, C. (Comp.) La aplicación de los tratados internacionales sobre derechos humanos por los tribunales locales.(p.283-350). Argentina: Del puerto,

Bacio, J. (2008). Corruption as a violation of human rights, The International Council on Human Rights Policy, Geneva: Editorial

Bryane, M. \& Habit, H. (2010). What Does Kosovo Teach Us about Using Human Rights Law to Prosecute Corruption Offences? En European Human Rights Law Review, 7.

Castejón, O. (2022). Venezuela: Universidad Rafael Urdaneta.CDESC (Por favor, nombre completo de esta sigla). (Año?). La aplicación interna del Pacto Internacional de Derechos Económicos, Sociales y Culturales. Observación General, 9.

CDESC Esta referencia se debe manejar igual a la anterior; por favor, corregir) El derecho a la educación, Observación General Número 13, 1999a.
CDESC; La no discriminación y los derechos económicos, sociales y culturales, Observación General Número 20, 2009.

CDESC; Planes de acción para la educación primaria, Observación General Número 11, 1999b.

CIPDH-ITESM; La Corrupción y los Derechos Humanos. Estableciendo el Vínculo, México, Consejo Internacional para las Políticas de Derechos Humanos e Instituto Tecnológico y de Estudios Superiores de Monterrey, 2009.

Courtis, C. (2008).La protección de los derechos económicos, sociales y culturales a través del artículo 26 de la Convención Americana sobre Derechos Humanos. En Ferrer, E. \& Zaldívar, A. La ciencia del derecho procesal constitucional. Estudios en homenaje a Héctor Fix-Zamudio en sus cincuenta años como investigador del derecho.(p.361-438). México: UNAM-Marcial Pons

Elorza, H. (2000). Estadística para las ciencias sociales y del comportamiento. México: C, Oxfor.

Freund, J. \& Simon, G. (1994). Estadística elemental. México: Pearson Prentice Hall. Goodwin, M. \& Rose-Sender, S. (2010). Linking Corruption and Human Rights: An Unwelcome Addition to the Development Discourse: Nederland: Tilburg University Legal Studies.

Gruenberg, C. (2007). Identificando posibles puntos de entrada para una alianza entre las estrategias de derechos humanos y anti-corrupción. Ginebtra:Consejo Internacional para el Estudio de los Derechos Humanos.

Hopkings, K., Hopkings, B. \&\& Glass, G. (1997). Estadística básica para las ciencias del comportamiento. México: A Simon \& Schuster Company.

Johnston, M. (2005). Es posible medir la corrupción ¿Pero podemos medir la reforma? En Revista Mexicana de Sociología, 2. 
Kumar, C. (2002). Corruption and human rights: The human right to corruption -free service- some constitutional and international perspectives. En India's National Magazine from the publishers of the Hindu 19 - Issue 19, September 14 - 27.

Moore, D. (2005). Estadística básica aplicada. España:Antoni Bosch.

Murray, R. \& Spiegel, L. (2002). Estadística. México: Mc Graw Hill.

Neville, T. (2005). Educational research: some basic concepts and terminology. Francia: International Institute for Educational Planning-UNESCO.

(2007). El Sistema de Tratados de Derechos Humanos de Naciones Unidas. Introducción a los tratados fundamentales de derechos humanos y a los órganos creados en virtud de tratados. Folleto Informativo, 30.

OCDE (2010). Resultados del Informe PISA 2009: Lo que los estudiantes saben y pueden hacer. Rendimiento de estudiantes en lectura, matemáticas y ciencias. España: Santillana.

Pilapitiya, T. (2004). The impact of corruption on the human rights based approach to development. United Nations Development Programme-Oslo Governance Centre-The Democratic Governance Fellowship Programme.

Posadas, A. (2006). Estado del arte en investigación sobre corrupción. En Primera Conferencia Internacional sobre Corrupción y Transparencia. Debatiendo las fronteras entre Estado, Mercado y Sociedad. México: Laboratorio de Documentación y Análisis de la Corrupción y la Transparencia del Instituto de Investigaciones Sociales de la Universidad Nacional Autónoma de México.

Rajagopal, B. Corruption, Legitimacy and Human Rights: The Dialectic of the Relationship. En Connecticut Journal of International Law, 14, 2.

Stephen, P. (2004). Education and corruption.
International Journal of educational Development, 24, 2004.

Tamez, P. (2003). La evaluación como factor de cambio en la educación. En Revista de información y análisis, 22.

Thuo, J. (2009). Defining the Relationship between Corruption and Human Rights. En University of Pennsylvania Journal of International Law, 31, 125.

Transparencia Internacional (2009a). Índice de Percepción de la Corrupción Breve nota metodológica. Extraído Febrero 8, 2013 desde http://www.transparencia.org.es/IPC\%C2\%B4s/ IPC $2009 /$ Metodolog \% C $3 \%$ AD a \% 20CPI_2009_short_methodology_spanish.pdf.

Transparencia Internacional (2009b). La corrupción amenaza la recuperación económica mundial y supone graves obstáculos para los países en conflicto. Extraído Febrero 14, 2013, desde http:/www.transparency.org/news/pressrelease/20091117_la_corrupcion_amenaza_la_recuperacion_economica_mundial.

Transparencia Internacional (2009c). Tabla de resultados 2009. Extraído Febrero 8, 2013 desde http://www.transparencia.org.es/INDICE\%20 DE\%20PERCEPCI\%C3\% 93N\%202009/ Tabla\%20sint\%C3\%A9tica.CPI_2009_table_ spanish.pdf.

Transparencia Internacional. (2008). Los derechos humanos y la corrupción. Documento de Trabajo núm. 5 Alemania: Transparencia Internacional.

UNESCO. (2004). (Por favor, poner nombre completo de la sigla) Education for all. The quality imperative. Francia: UNESCO.

UNICEF(Por favor, poner nombre completo de la sigla).Defining Quality in Education. USA: UNICEF.

Vincent, R. (2008).Corruption in Education: A Global Legal Challenge. Santa Clara L. Rev. 1, 48. 